\title{
Flavor Decomposition of the Sea Quark Helicity Distributions in the Nucleon from Semi-inclusive Deep-inelastic Scattering
}

A. Airapetian, ${ }^{30}$ N. Akopov, ${ }^{30}$ Z. Akopov, ${ }^{30}$ M. Amarian, ${ }^{6,30}$ V.V. Ammosov, ${ }^{22}$ A. Andrus, ${ }^{15}$ E.C. Aschenauer, ${ }^{6}$ W. Augustyniak,${ }^{29}$ R. Avakian ${ }^{30}$ A. Avetissian, ${ }^{30}$ E. Avetissian,${ }^{10}$ P. Bailey,${ }^{15}$ V. Baturin,${ }^{21}$ C. Baumgarten, ${ }^{19}$ M. Beckmann, ${ }^{5}$ S. Belostotski, ${ }^{21}$ S. Bernreuther ${ }^{27}$ N. Bianchi, ${ }^{10}$ H.P. Blok,${ }^{20,}{ }^{28}$ H. Böttcher,${ }^{6}$ A. Borissov, ${ }^{17}$ M. Bouwhuis, ${ }^{15}$ J. Brack, ${ }^{4}$ A. Brüll, ${ }^{16}$ V. Bryzgalov, ${ }^{22}$ G.P. Capitani, ${ }^{10}$ H.C. Chiang, ${ }^{15}$ G. Ciullo, ${ }^{9}$ M. Contalbrigo, ${ }^{9}$ P.F. Dalpiaz,${ }^{9}$ R. De Leo,${ }^{3}$ L. De Nardo, ${ }^{1}$ E. De Sanctis,${ }^{10}$ E. Devitsin,${ }^{18}$ P. Di Nezza,${ }^{10}$ M. Düren,${ }^{13}$ M. Ehrenfried,${ }^{8}$ A. Elalaoui-Moulay ${ }^{2}$ G. Elbakian ${ }^{30}$ F. Ellinghaus,${ }^{6}$ U. Elschenbroich, ${ }^{12}$ J. Ely, ${ }^{4}$ R. Fabbri, ${ }^{9}$ A. Fantoni, ${ }^{10}$ A. Fechtchenko, ${ }^{7}$ L. Felawka,${ }^{26}$ B. Fox,${ }^{4}$ J. Franz,${ }^{11}$ S. Frullani, ${ }^{24}$ Y. Gärber,${ }^{8}$ G. Gapienko, ${ }^{22}$ V. Gapienko ${ }^{22}$ F. Garibaldi, ${ }^{24}$ K. Garrow,${ }^{1,25}$ E. Garutti ${ }^{20}$ D. Gaskell,${ }^{4}$ G. Gavrilov $,{ }^{5}, 26$ V. Gharibyan, ${ }^{30}$ G. Graw, ${ }^{19}$ O. Grebeniouk, ${ }^{21}$ L.G. Greeniaus, ${ }^{1,26}$ K. Hafidi, ${ }^{2}$ M. Hartig,${ }^{26}$ D. Hasch, ${ }^{10}$ D. Heesbeen, ${ }^{20}$ M. Henoch,${ }^{8}$ R. Hertenberger, ${ }^{19}$ W.H.A. Hesselink,${ }^{20,}{ }^{28}$ A. Hillenbrand,${ }^{8}$ M. Hoek, ${ }^{13}$ Y. Holler,${ }^{5}$ B. Hommez, ${ }^{12}$ G. Iarygin, ${ }^{7}$ A. Ivanilov, ${ }^{22}$ A. Izotov,${ }^{21}$ H.E. Jackson, ${ }^{2}$ A. Jgoun, ${ }^{21}$ R. Kaiser ${ }^{14}$ E. Kinney, ${ }^{4}$ A. Kisselev, ${ }^{21}$ K. Königsmann, ${ }^{11}$ M. Kopytin,${ }^{6}$ V. Korotkov,${ }^{6}$ V. Kozlov,${ }^{18}$ B. Krauss, ${ }^{8}$ V.G. Krivokhijine, ${ }^{7}$ L. Lagamba,${ }^{3}$ L. Lapikás,${ }^{20}$ A. Laziev, ${ }^{20,28}$ P. Lenisa, ${ }^{9}$ P. Liebing, ${ }^{6}$ T. Lindemann, ${ }^{5}$ K. Lipka, ${ }^{6}$ W. Lorenzon, ${ }^{17}$ J. Lu, ${ }^{26}$ B. Maiheu, ${ }^{12}$ N.C.R. Makins,${ }^{15}$ B. Marianski,${ }^{29}$ H. Marukyan,${ }^{30}$ F. Masoli,${ }^{9}$ V. Mexner,${ }^{20}$ N. Meyners,${ }^{5}$ O. Mikloukho, ${ }^{21}$ C.A. Miller, ${ }^{1,}{ }^{26}$ Y. Miyachi, ${ }^{27}$ V. Muccifora,${ }^{10}$ A. Nagaitsev,${ }^{7}$ E. Nappi,${ }^{3}$ Y. Naryshkin, ${ }^{21}$ A. Nass, ${ }^{8}$ M. Negodaev,${ }^{6}$ W.-D. Nowak,${ }^{6}$ K. Oganessyan,${ }^{5,10}$ H. Ohsuga, ${ }^{27}$ G. Orlandi, ${ }^{24}$ N. Pickert,${ }^{8}$ S. Potashov, ${ }^{18}$ D.H. Potterveld,${ }^{2}$

M. Raithel,${ }^{8}$ D. Reggiani, ${ }^{9}$ P.E. Reimer, ${ }^{2}$ A. Reischl, ${ }^{20}$ A.R. Reolon,${ }^{10}$ C. Riedl,${ }^{8}$ K. Rith, ${ }^{8}$ G. Rosner,${ }^{14}$

A. Rostomyan, ${ }^{30}$ L. Rubacek, ${ }^{13}$ D. Ryckbosch, ${ }^{12}$ Y. Salomatin, ${ }^{22}$ I. Sanjiev, ${ }^{2,21}$ I. Savin, ${ }^{7}$ C. Scarlett, ${ }^{17}$ A. Schäfer, ${ }^{23}$ C. Schill, ${ }^{11}$ G. Schnell, ${ }^{6}$ K.P. Schüler, ${ }^{5}$ A. Schwind, ${ }^{6}$ J. Seele, ${ }^{15}$ R. Seidl, ${ }^{8}$ B. Seitz, ${ }^{13}$ R. Shanidze,${ }^{8}$ C. Shearer ${ }^{14}$ T.-A. Shibata, ${ }^{27}$ V. Shutov,${ }^{7}$ M.C. Simani, ${ }^{20,}{ }^{28}$ K. Sinram,${ }^{5}$ M. Stancari,${ }^{9}$ M. Statera, ${ }^{9}$ E. Steffens, ${ }^{8}$ J.J.M. Steijger, ${ }^{20}$ J. Stewart,${ }^{6}$ U. Stösslein,${ }^{4}$ P. Tait,${ }^{8}$ H. Tanaka, ${ }^{27}$ S. Taroian, ${ }^{30}$ B. Tchuiko,${ }^{22}$ A. Terkulov, ${ }^{18}$ A. Tkabladze,${ }^{6}$ A. Trzcinski, ${ }^{29}$ M. Tytgat,${ }^{12}$ A. Vandenbroucke, ${ }^{12}$ P. van der Nat, ${ }^{20,}{ }^{28}$ G. van der Steenhoven, ${ }^{20}$

M.C. Vetterli, ${ }^{25}{ }^{26}$ V. Vikhrov, ${ }^{21}$ M.G. Vincter, ${ }^{1}$ J. Visser,${ }^{20}$ C. Vogel,${ }^{8}$ M. Vogt,${ }^{8}$ J. Volmer, ${ }^{6}$ C. Weiskopf,${ }^{8}$ J. Wendland, ${ }^{25,}{ }^{26}$ J. Wilbert, ${ }^{8}$ G. Ybeles Smit, ${ }^{28}$ S. Yen, ${ }^{26}$ B. Zihlmann, ${ }^{20,}{ }^{28}$ H. Zohrabian, ${ }^{30}$ and P. Zupranski ${ }^{29}$

(The Hermes Collaboration)

\author{
${ }^{1}$ Department of Physics, University of Alberta, Edmonton, Alberta T6G 2J1, Canada \\ ${ }^{2}$ Physics Division, Argonne National Laboratory, Argonne, Illinois 60439-4843, USA \\ ${ }^{3}$ Istituto Nazionale di Fisica Nucleare, Sezione di Bari, 70124 Bari, Italy \\ ${ }^{4}$ Nuclear Physics Laboratory, University of Colorado, Boulder, Colorado 80309-0446, USA \\ ${ }^{5}$ DESY, Deutsches Elektronen-Synchrotron, 22603 Hamburg, Germany \\ ${ }^{6}$ DESY Zeuthen, 15738 Zeuthen, Germany \\ ${ }^{7}$ Joint Institute for Nuclear Research, 141980 Dubna, Russia \\ ${ }^{8}$ Physikalisches Institut, Universität Erlangen-Nürnberg, 91058 Erlangen, Germany \\ ${ }^{9}$ Istituto Nazionale di Fisica Nucleare, Sezione di Ferrara and \\ Dipartimento di Fisica, Università di Ferrara, 44100 Ferrara, Italy \\ ${ }^{10}$ Istituto Nazionale di Fisica Nucleare, Laboratori Nazionali di Frascati, 00044 Frascati, Italy \\ ${ }^{11}$ Fakultät für Physik, Universität Freiburg, 79104 Freiburg, Germany \\ ${ }^{12}$ Department of Subatomic and Radiation Physics, University of Gent, 9000 Gent, Belgium \\ ${ }^{13}$ Physikalisches Institut, Universität Gießen, 35392 Gießen, Germany \\ ${ }^{14}$ Department of Physics and Astronomy, University of Glasgow, Glasgow G12 8QQ, United Kingdom \\ ${ }^{15}$ Department of Physics, University of Illinois, Urbana, Illinois 61801-3080, USA \\ ${ }^{16}$ Laboratory for Nuclear Science, Massachusetts Institute of Technology, Cambridge, Massachusetts 02139, USA \\ ${ }^{17}$ Randall Laboratory of Physics, University of Michigan, Ann Arbor, Michigan 48109-1120, USA \\ ${ }^{18}$ Lebedev Physical Institute, 117924 Moscow, Russia \\ ${ }^{19}$ Sektion Physik, Universität München, 85748 Garching, Germany \\ ${ }^{20}$ Nationaal Instituut voor Kernfysica en Hoge-Energiefysica (NIKHEF), 1009 DB Amsterdam, The Netherlands \\ ${ }^{21}$ Petersburg Nuclear Physics Institute, St. Petersburg, Gatchina, 188350 Russia \\ ${ }^{22}$ Institute for High Energy Physics, Protvino, Moscow region, 142281 Russia \\ ${ }^{23}$ Institut für Theoretische Physik, Universität Regensburg, 93040 Regensburg, Germany \\ ${ }^{24}$ Istituto Nazionale di Fisica Nucleare, Sezione Roma 1, Gruppo Sanità \\ and Physics Laboratory, Istituto Superiore di Sanità, 00161 Roma, Italy \\ ${ }^{25}$ Department of Physics, Simon Fraser University, Burnaby, British Columbia V5A 1S6, Canada \\ ${ }^{26}$ TRIUMF, Vancouver, British Columbia V6T 2A3, Canada \\ ${ }^{27}$ Department of Physics, Tokyo Institute of Technology, Tokyo 152, Japan
}




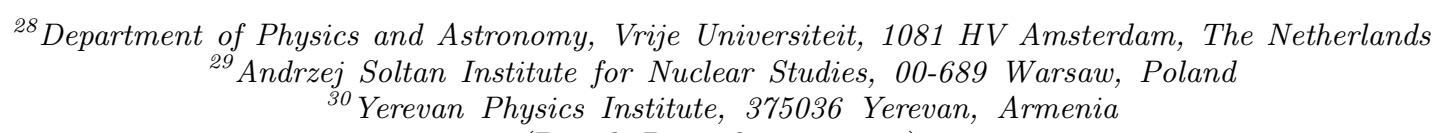

(Dated: December 25, 2013)

\begin{abstract}
Double-spin asymmetries of semi-inclusive cross sections for the production of identified pions and kaons have been measured in deep-inelastic scattering of polarized positrons on a polarized deuterium target. Five helicity distributions including those for three sea quark flavors were extracted from these data together with re-analyzed previous data for identified pions from a hydrogen target. These distributions are consistent with zero for all three sea flavors. A recently predicted flavor asymmetry in the polarization of the light quark sea appears to be disfavored by the data.
\end{abstract}

PACS numbers: 13.60.-r, 13.88.+e, 14.20.Dh, 14.65.-q

The quark-parton picture of nucleon structure includes the three valence quarks that define the quantum numbers of the bound state, gluons that mediate the strong force between the quarks, as well as a significant presence of virtual sea quarks from gluon splitting and nonperturbative processes. The relativistic motion of these bound partons has been thoroughly studied, mainly in deeply inelastic scattering (DIS) of leptons [1]. However, the photon exchange that dominates the interactions of charged leptons limits their flavor sensitivity to the magnitude of the quark charge, failing to distinguish sea quarks. The parity-violating charged-current interaction present in neutrino scattering and $\mathrm{W}^{ \pm}$production helps to distinguish the parton distribution functions (PDFs) $q(x)$ of quarks and antiquarks of flavors $q=($ up, down, strange, charm) 2, 3]. Here $x$ is the dimensionless Bjorken scaling variable representing the momentum fraction of the target carried by the parton in the frame where the target has "infinite" momentum.

These PDFs depend on whether the parton's helicity is equal or opposite to that of the nucleon. The differences, or helicity distributions, $\Delta q(x)=q^{\uparrow \uparrow}(x)-q^{\uparrow \downarrow}(x)$ are much less well known, not only because of the far more limited data set for scattering of polarized charged leptons on polarized targets, but also because polarized targets are presently impractical with neutrino beams. A standard approach is to further constrain the problem using the different flavor sensitivity of hyperon $\beta$ decay data, via the additional assumption of SU(3) flavor symmetry among the structures of the octet baryons. The first such analysis 15 years ago [4] revealed the celebrated "proton spin puzzle" - an apparent cancellation among all the $\Delta q$ 's to make a small net contribution to the spin $1 / 2$ of the nucleon. Also, the strange sea polarization appeared to be negative. These findings have since been confirmed with steadily improving precision [5].

An alternative approach avoids this assumption about SU(3) symmetry by extracting more information from the DIS data. A quark that absorbs an energetic virtual photon gives rise to a "jet" of final-state hadrons, the composition of which reflects the flavor of the struck quark. At the moderate energies of present fixed-target measurements where few hadrons are produced, individ- ual hadrons from the fragmentation of the struck quark can also serve to "tag" its flavor. This exploitation of hadrons detected with the scattered leptons in such semiinclusive measurements requires knowledge of the probabilities of the various types $h$ of hadrons emerging from a struck quark of a given flavor $q$. These probabilities are embodied in the fragmentation functions $D_{q}^{h}(z)$, where $z \equiv E_{h} / \nu$ and $\nu$ and $E_{h}$ are the energies in the target rest frame of the absorbed virtual photon (and hence of the struck quark) and the detected hadron. Although fragmentation functions have been extracted from mostly high energy $e^{+} e^{-}$collider data [6, 7], their applicability at lower energies is supported by their agreement with fragmentation functions [8] for charged pions extracted from HERMES measurements of hadron multiplicities, and also by the agreement between neutral pion multiplicities measured at widely different energies $[9]$.

Given an adequate understanding of the fragmentation process, a complete flavor decomposition of the quark and antiquark helicity distributions can be extracted from sufficiently precise measurements of double-spin asymmetries in the cross sections for leptoproduction of various types of hadrons. In leading order (LO), the semiinclusive virtual photo-absorption cross section for production of a hadron of type $h$ takes the factorized form

$$
\sigma^{h}(x, z) \propto \sum_{q} e_{q}^{2} q(x) D_{q}^{h}(z) .
$$

The sum at the moderate beam energies considered here is over quark and antiquark flavors $q=(u, \bar{u}, d, \bar{d}, s, \bar{s})$, and $e_{q}$ is the quark charge. For simplicity, we suppress here the weak logarithmic dependence of all functions on the spatial resolution scale corresponding to $-Q^{2}$, the squared four-momentum of the exchanged virtual photon. The double-spin asymmetry $A_{1}^{h}$ in the above cross section is then given by

$$
A_{1}^{h}(x, z)=\frac{\sum_{q} e_{q}^{2} \Delta q(x) D_{q}^{h}(z)}{\sum_{q} e_{q}^{2} q(x) D_{q}^{h}(z)} \frac{(1+R(x))}{\left(1+\gamma^{2}\right)},
$$

where the factor involving $\gamma^{2}=Q^{2} / \nu^{2}$ and $R$, the ratio of longitudinal to transverse virtual photon cross sections, accounts for the longitudinal component included 
in most parametrizations of the unpolarized PDFs $q(x)$. With asymmetry data available for a variety of hadrons from both proton and "neutron" targets, the above system of equations becomes sufficiently constrained to be solved to extract the $\Delta q(x)$ for several flavors $q$. Two such analyses have been reported [10, 11], using undifferentiated hadrons. The limited statistical precision of those asymmetry data required severe symmetry constraints to be applied among the polarizations of the sea quark flavors. Here we report much more precise asymmetry data for identified pions and kaons from a deuteron target recorded by HERMES in 1998-2000. In combination with identified pions from hydrogen in 1996-1997 and with inclusive data, they result in the first extraction of quark polarizations for five independent flavors from semi-inclusive data. Sea quarks of all three flavors are treated independently, although in the strange sector, only $\Delta s(x)$ could be extracted and not $\Delta \bar{s}(x)$.

Asymmetries sensitive to the helicity distributions require both the beam and target to be polarized. A unique feature of the Hermes experiment is its target of pure nuclear-polarized atomic gas quasi-confined in an openended $40 \mathrm{~cm}$ long cylindrical storage cell, through which passes the $E=27.6 \mathrm{GeV}$ electron/positron beam of the HERA storage ring at DESY. The self-induced beam polarization is measured continuously by two independent polarimeters using Compton backscattering of circularly polarized laser light 12, 13]. The average beam polarization for the deuteron (proton) data set was $0.532(0.530)$ with a fractional systematic uncertainty of $1.9 \%(3.4 \%)$. The target cell is fed by an atomic-beam source based on Stern-Gerlach separation [14] with hyperfine transitions. The nuclear polarization of the atoms is flipped at $90 \mathrm{~s}$ time intervals, while both this polarization and the atomic fraction inside the target cell are continuously measured [15, 16]. The average value of the deuteron (proton) polarization was $0.844(0.824)$ with a fractional systematic uncertainty of $4.4(4.2) \%$.

Scattered beam leptons and any coincident hadrons are detected by the HERMES spectrometer 17]. Leptons are identified with an efficiency exceeding $98 \%$ and a hadron contamination of less than $1 \%$ using an electromagnetic calorimeter, a transition-radiation detector, a preshower scintillation counter and a Čerenkov detector. Another unique feature of the experiment is the complete hadron identification provided for the deuterium data set by a dual-radiator ring-imaging Čerenkov detector [18]. Only pions were identified by a threshold-Čerenkov detector during the earlier hydrogen measurements.

Events were selected subject to the kinematic requirements $Q^{2}>1 \mathrm{GeV}^{2}, W^{2}>10 \mathrm{GeV}^{2}$ and $y<0.85$, where $W$ is the invariant mass of the photon-nucleon system, and $y=\nu / E$. Coincident hadrons were accepted if $0.2<z<0.8$ and $x_{F} \approx 2 p_{L} / W>0.1$, where $p_{L}$ is the longitudinal momentum of the hadron with respect to the virtual photon direction in the photon-nucleon center of mass frame. The limit on $x_{F}$ suppressed most of the contamination of the hadron sample by target fragmentation, as evidenced by the consistency of all the $\Delta q$ 's extracted in $0.45<z<0.8$ with those from $0.2<z<0.45$ where target fragmentation is more likely to appear, and by the negligible effect on any $\Delta q(x)$ in the range $x<0.3$ of excluding inclusive asymmetries from the analysis.

The (semi-)inclusive virtual photo-absorption asymmetries $A_{1}^{h}$ were derived from the lepton scattering asymmetries $A_{\|}^{h}$ using the relation $A_{1}^{h}=A_{\|}^{h} /[D(1+\gamma \eta)]$, where $D$ is the depolarization factor for the virtual photon and $\eta$ is a kinematic factor [19]. The effects of internal QED radiation and instrumental resolution were simulated 20, 21, 22], and corrections were applied to $A_{\|}^{h}$ using a technique that unfolds kinematic migration of events 23. This results in small statistical correlations between bins in $x$, which were incorporated in the subsequent analysis. Small corrections were applied for the combined effect of the dependences on the hadron azimuthal angle of the spectrometer acceptance and of the unpolarized cross section.

The corrected semi-inclusive asymmetries for the deuterium target are shown in Fig. 1 They represent the first such results for identified pions and kaons. The proton and inclusive deuteron asymmetries as well as more details about the analysis can be found in Ref. [24]. The asymmetries for the proton from 1996-1997 differ within the systematic uncertainties from the published values 11] due only to refinements in the analysis such as those described above 24]. The contributions to the systematic uncertainties on $A_{1}^{h}$ include those from the beam and target polarizations, estimates of those due to the effects of the spectrometer acceptance, which were studied using the PePSi/Jetset DIS Monte Carlo event generator [20, 22] with the photon-gluon fusion and QCDCompton processes enabled, a contribution from the ratio $R$ [25], and an estimate of the neglected small effect of the spin structure function $\mathrm{g}_{2}(x)$ [26] representing interference of longitudinal and transverse photons.

Integrating Eq. (1) over $z$, Eq. (2) becomes

$$
A_{1}^{h}(x)=\sum_{q} P_{q}^{h}(x) \frac{\Delta q(x)}{q(x)} \frac{(1+R(x))}{\left(1+\gamma^{2}\right)},
$$

where $P_{q}^{h}(x)$ are the spin-independent purities:

$$
P_{q}^{h}(x) \equiv \frac{e_{q}^{2} q(x) \int_{0.2}^{0.8} D_{q}^{h}(z) d z}{\sum_{q^{\prime}} e_{q^{\prime}}^{2} q^{\prime}(x) \int_{0.2}^{0.8} D_{q^{\prime}}^{h}(z) d z} .
$$

Each purity function describes the conditional probability that the virtual photon hit a quark of flavor $q$ when a hadron of type $h$ was detected. In the inclusive case, $P_{q}^{h}$ is replaced by $P_{q}(x)=e_{q}^{2} q(x) / \sum_{q^{\prime}} e_{q^{\prime}}^{2} q^{\prime}(x)$. In analogy with Eq. (4), the purities used in this analysis were extracted from the above-mentioned Monte Carlo simulation (but now including only the effects of 


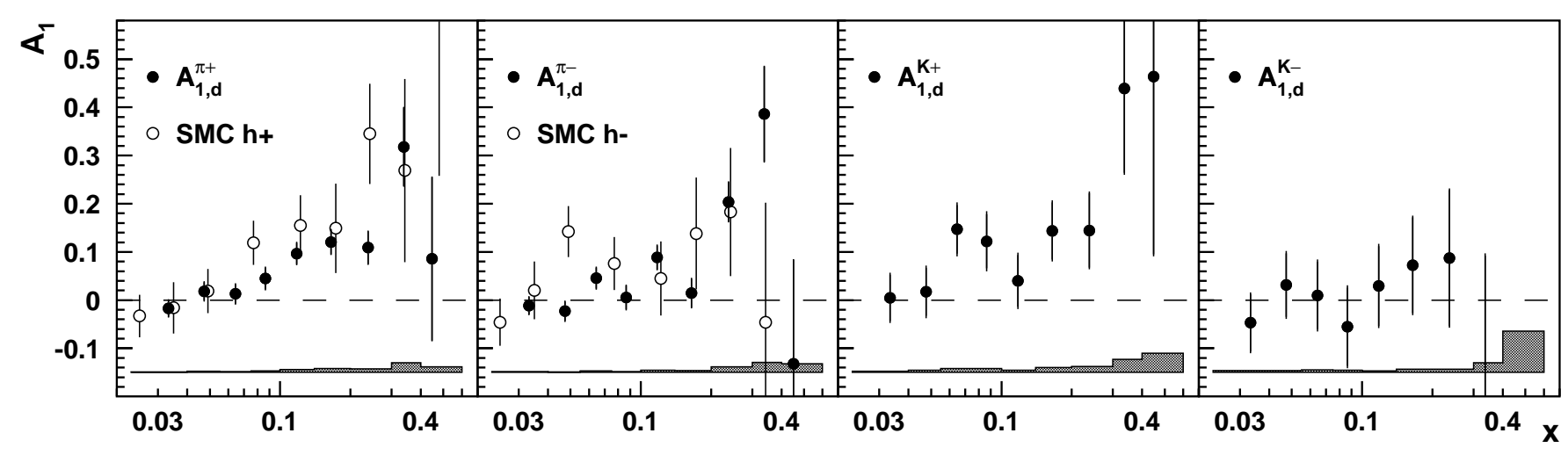

FIG. 1: Virtual photo-absorption asymmetries $A_{1}^{h}$ for semi-inclusive DIS on the deuterium target as a function of Bjorken $x$, for identified charged pions (compared to all charged hadrons from SMC [10] in the $x$-range of the present experiment), and for identified charged kaons. The error bars are statistical, and the bands at the bottom represent the systematic uncertainties. Two data points for $\mathrm{K}^{-}$at large $x$ are off-scale with large error bars.

geometric acceptance and not those of QED radiation and detector resolution) as $P_{q}^{h}(x)=N_{q}^{h}(x) / \sum_{q^{\prime}} N_{q^{\prime}}^{h}(x)$, where $N_{q}^{h}$ is the number of hadrons of type $h$, in the geometric experimental acceptance and in the interval $0.2<z<0.8$, that were produced when a quark of flavor $q$ was struck. The simulation employs the CTEQ5L leading order parametrization [2] for the unpolarized PDFs, and JETSET fragmentation parameters that were tuned to approximate hadron multiplicities measured at HERMES 24]. Nuclear corrections for the deuteron target were applied, based on a D-state probability $\omega_{D}=0.05 \pm 0.01[27$.

Eq. (3) can be written in matrix form

$$
\vec{A}(x)=\mathcal{P}(x) \cdot \vec{Q}(x),
$$

where the measured asymmetries are elements of the vector $\vec{A}(x)=\left(A_{1 p}, A_{1 p}^{\pi^{+}}, A_{1 p}^{\pi^{-}}, A_{1 d}, A_{1 d}^{\pi^{+}}, A_{1 d}^{\pi^{-}}, A_{1 d}^{\mathrm{K}^{+}}, A_{1 d}^{\mathrm{K}^{-}}\right)$. The matrix $\mathcal{P}$ contains the purities for the proton and deuteron, while the vector $\vec{Q}(x)$ contains the quark and antiquark polarizations:

$\vec{Q}(x)=\left(\frac{\Delta u}{u}, \frac{\Delta d}{d}, \frac{\Delta \bar{u}}{\bar{u}}, \frac{\Delta \bar{d}}{\bar{d}}, \frac{\Delta s}{s}, \frac{\Delta \bar{s}}{\bar{s}} \equiv 0 \pm \frac{1}{\sqrt{3}}\right)$.

The set of Eq. (5) evaluated in all $x$-bins is solved together for the vector $\vec{Q}$ by $\chi^{2}$-minimisation, accounting for the correlations between $x$-bins and between the various asymmetries. For $x>0.3$ the sea polarizations are set to zero, and the effect on the non-sea polarizations of varying these sea polarizations by $\pm 1 / \sqrt{3}$ (the standard deviation of a distribution uniform over \pm 1 ) is included in their systematic uncertainties. A contribution from a similar variation of $[\Delta \bar{s} / \bar{s}](x)$ is applied for all $x$.

Finally, the $\Delta q(x)$ results are determined as the products of the extracted polarizations $[\Delta q / q](x)$ and the unpolarized PDFs from Ref. 2] at the mean scale $\left\langle Q^{2}\right\rangle=$ $2.5 \mathrm{GeV}^{2}$ of the present work. It is assumed that the polarizations are independent of $Q^{2}$ within the $Q^{2}$ range of this measurement. The systematic uncertainties on the $\Delta q(x)$ include contributions from the $A_{1}^{h}$ data and those from fragmentation, which were obtained by comparing the results from two sets of fragmentation parameters in JETSET. Fig. 2 shows the results compared to two LO QCD fits 28, 29] to all available inclusive data. These fits assume not only $\mathrm{SU}(3)$ flavor symmetry to incorporate hyperon beta decay data, but also explicit symmetry among the three sea quark distributions.

The extracted distributions $\Delta u(x)$ and $\Delta d(x)$ are consistent with previous (semi-)inclusive results 10, 11], but have much improved precision. The sea distributions, extracted separately here for the first time, are consistent with zero and with each other. The strange sea was previously found to be negatively polarized in the analysis of only inclusive data assuming SU(3) symmetry applied to hyperon beta decay data. However, the first moments from such analyses evaluated over the measured $x$-range $(\Delta s+\Delta \bar{s}) / 2 \equiv \int_{0.023}^{0.3} \Delta s(x) \mathrm{d} x$ are typically not in disagreement with that partial moment of the density extracted here: $\Delta s=+0.03 \pm 0.03$ (stat.) \pm 0.01 (syst.).

Fig. [3] shows the flavor asymmetry $\Delta \bar{u}(x)-\Delta \bar{d}(x)$ in the light quark sea in comparison with the prediction of a theoretical calculation based on the chiral quark soliton model [30]. This model is an effective field theory embodying fundamental features of QCD that successfully describes a large body of baryon properties 31]. For example, it explains the previously observed substantial value for the unpolarized flavour asymmetry $\bar{u}(x)-\bar{d}(x)$ 32. Its prediction for the polarized moment is $\Delta \bar{u}-\Delta \bar{d}=+0.21 \pm 0.05$, to be compared to the present experimental value of $+0.05 \pm 0.06$ (stat.) \pm 0.03 (syst.). The difference is about $2 \sigma$, when combining all uncertainties in quadrature. Again both moments are evaluated over only the measured range.

In conclusion, a purity-based extraction from new semi-inclusive DIS data has produced separated helicity distributions for five flavors: $\Delta u, \Delta d, \Delta \bar{u}, \Delta \bar{d}$, and $\Delta s$. 


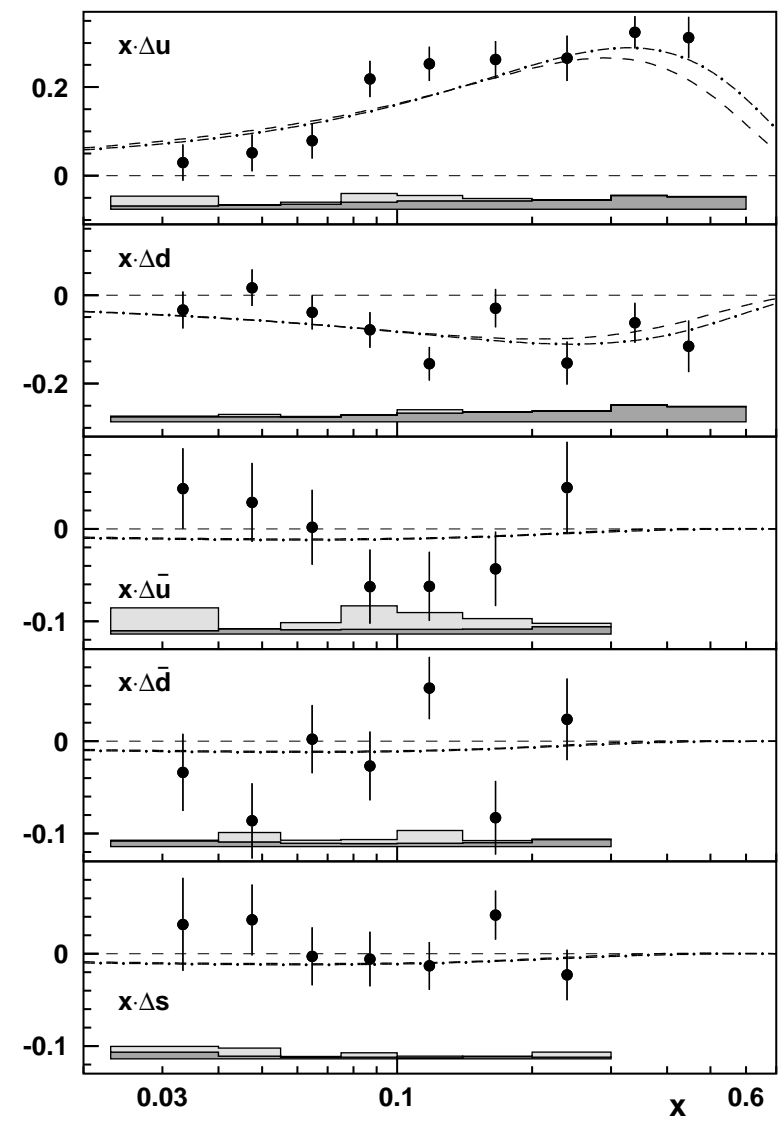

FIG. 2: Quark helicity distributions at $\left\langle Q^{2}\right\rangle=2.5 \mathrm{GeV}^{2}$, as a function of Bjorken $x$, compared to two LO QCD fits to previously published inclusive data shown as dashed [28] ("standard scenario") and dot-dashed [29] ("scenario 1") curves. The error bars are statistical and the bands at the bottom represent the systematic uncertainties, where the light area is the contribution due to the uncertainties of the fragmentation model, and the dark area is the contribution due to those of the asymmetries.

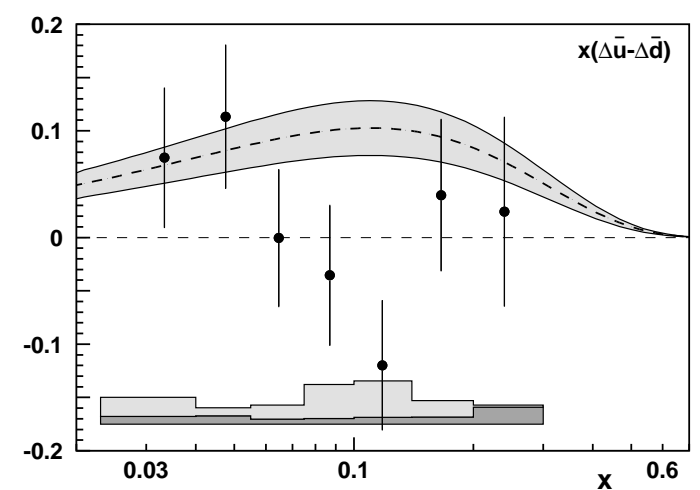

FIG. 3: The light quark sea flavor asymmetry $x \cdot(\Delta \bar{u}-\Delta \bar{d})$ in the helicity distributions, at $\left\langle Q^{2}\right\rangle=2.5 \mathrm{GeV}^{2}$, compared to a theoretical prediction [30] (dashed curve with theoretical uncertainty band). The experimental error bars and bands have the same meaning as in Fig. 2
The polarized densities for all sea flavors are found to be consistent with zero.

We gratefully acknowledge the DESY management for its support, the HERA team for providing the polarised beams for HERMES, the collaborating institutions for their significant effort, and our funding agencies for financial support.

[1] A. Thomas and W. Weise, The Structure of the Nucleon (Wiley-VCH, 2001).

[2] H. L. Lai et al., Eur. Phys. J. C 12, 375 (2000).

[3] A. D. Martin et al., Eur. Phys. J. C 23, 73 (2002).

[4] J. Ashman et al. (EMC), Phys. Lett. B206, 364 (1988).

[5] B. Adeva et al. (SMC), Phys. Rev. D 58, 112002 (1998).

[6] B. A. Kniehl et al., Nucl. Phys. B582, 514 (2000).

[7] S. Kretzer, Phys. Rev. D 62, 054001 (2000).

[8] S. Kretzer et al., Eur. Phys. J. C 22, 269 (2001).

[9] A. Airapetian et al. (Hermes), Eur. Phys. J. C 21, 599 (2001).

[10] B. Adeva et al. (SMC), Phys. Lett. B420, 180 (1998).

[11] K. Ackerstaff et al. (Hermes), Phys. Lett. B464, 123 (1999).

[12] D. P. Barber et al., Nucl. Inst. \& Meth. A 338, 166 (1994).

[13] M. Beckmann et al., Nucl. Inst. \& Meth. A 479, 334 (2002).

[14] F. Stock et al., Nucl. Inst. \& Meth. A 343, 334 (1994).

[15] C. Baumgarten et al., Nucl. Inst. \& Meth. A 482, 606 (2002).

[16] C. Baumgarten et al., Nucl. Inst. \& Meth. A 496, 263 (2003).

[17] K. Ackerstaff et al. (Hermes), Nucl. Inst. \& Meth. A 417, 230 (1998).

[18] N. Akopov et al., Nucl. Inst. \& Meth. A 479, 511 (2002).

[19] A. Airapetian et al. (Hermes), Phys. Lett. B442, 484 (1998).

[20] L. Mankiewicz et al., Comp. Phys. Comm. 71, 305 (1992).

[21] I. Akushevich et al. (1998), hep-ph/9906408.

[22] T. Sjöstrand et al., Comp. Phys. Comm. 135, 238 (2001).

[23] C. A. Miller (in preparation).

[24] A. Airapetian et al. (Hermes) (in preparation).

[25] K. Abe et al. (E143), Phys. Lett. B452, 194 (1999).

[26] P. L. Anthony et al. (E155), Phys. Lett. B553, 18 (2003).

[27] B. Desplanques, Phys. Lett. B203, 200 (1988).

[28] M. Glück et al., Phys. Rev. D 63, 094005 (2001).

[29] J. Blümlein and H. Böttcher, Nucl. Phys. B636, 225 (2002).

[30] B. Dressler et al., Eur. Phys. J. C 14, 147 (2000).

[31] C. V. Christov et al., Prog. Part. Nucl. Phys. 37, 91 (1996).

[32] B. Dressler et al. (1998), hep-ph/9809487. 